\title{
Nonreciprocal Green's function retrieval by cross correlation
}

\author{
Kees Wapenaar \\ Department of Geotechnology, Delft University of Technology, P.O. Box 5028, 2600 GA Delft, The Netherlands \\ c.p.a.wapenaar@tudelft.nl
}

\begin{abstract}
The cross correlation of two recordings of a diffuse acoustic wave field at different receivers yields the Green's function between these receivers. In nearly all cases considered so far the wave equation obeys timereversal invariance and the Green's function obeys source-receiver reciprocity. Here the theory is extended for nonreciprocal Green's function retrieval in a moving medium. It appears that the cross correlation result is asymmetric in time. The causal part represents the Green's function from one receiver to the other whereas the acausal part represents the time-reversed version of the Green's function along the reverse path.

(C) 2006 Acoustical Society of America

PACS numbers: 43.20.Bi, 43.60.Ac, 43.60.Tj [ANN]

Date Received: March 12, 2006 Date Accepted: June 13, 2006
\end{abstract}

\section{Introduction}

It has been shown by many researchers in geophysics, ultrasonics, and underwater acoustics that the cross correlation of acoustic wave fields recorded by two different receivers yields the response at one of the receiver positions as if there was a source at the other. ${ }^{1-7}$ Various theories have been developed to explain this phenomenon, ranging from diffusion theory for enclosures ${ }^{8,9}$ multiple scattering theory and stationary phase theory for random media, ${ }^{10-12}$ and reciprocity theory for deterministic and random media. ${ }^{13-15}$ In nearly all cases it is assumed that the medium is lossless and nonmoving, which is equivalent with assuming that the underlying wave equation is invariant for time reversal. Moreover, in all cases the Green's functions obey source-receiver reciprocity. The time-reversal invariance together with the source-receiver reciprocity property has been elegantly exploited in an intuitive derivation, ${ }^{16}$ building on earlier work on time-reversed acoustic focusing. ${ }^{17}$ In a medium with losses the wave equation is no longer invariant for time reversal, but, as long as the medium is not moving, source-receiver reciprocity still holds. When the losses are not too high, the cross-correlation method yields a Green's function with correct travel times and approximate amplitudes. ${ }^{18,19}$ On the other hand, in a moving medium, both the time-reversal invariance and source-receiver reciprocity break down. It has previously been shown that, with some modifications, time-reversed acoustic focusing can still work in a moving medium. ${ }^{20,21}$ In this paper we derive a theory for nonreciprocal Green's function retrieval by cross correlation in a moving medium.

\section{Nonreciprocal Green's function representation}

The basis for our derivation is a reciprocity theorem, where "reciprocity" should be interpreted in a broader sense than source-receiver reciprocity. In general a reciprocity theorem relates two independent acoustic states in one and the same domain. ${ }^{22,23}$ One can distinguish between reciprocity theorems of the convolution type and of the correlation type. ${ }^{24}$ In the following we derive a correlation-type reciprocity theorem for a moving, arbitrary inhomogeneous, lossless acoustic medium, and show step-by-step how this leads to a simple expression for nonreciprocal Green's function retrieval by cross correlation.

Consider an acoustic wave field, characterized by the acoustic pressure $p(\mathbf{x}, t)$ and particle velocity $v_{i}(\mathbf{x}, t)$, propagating in a lossless inhomogeneous flowing medium with mass density $\rho(\mathbf{x})$, compressibility $\kappa(\mathbf{x})$, and stationary inhomogeneous flow velocity $v_{k}^{0}(\mathbf{x})$. Throughout this paper we assume that the spatial variations of the flow velocity are small in 
comparison with those of the wave field. For this situation the equation of motion and the stressstrain relation read $\rho D_{t} v_{i}+\partial_{i} p=0$ and $\kappa D_{t} p+\partial_{i} v_{i}=q$, respectively, where $q(\mathbf{x}, t)$ is a source distribution in terms of volume injection rate density, $\partial_{i}$ is the partial derivative in the $x_{i}$ direction, and $D_{t}$ is the material time derivative, ${ }^{25}$ defined as $D_{t}=\partial_{t}+v_{k}^{0} \partial_{k}$. We define the temporal Fourier transform of a space- and time-dependent quantity $p(\mathbf{x}, t)$ as $\hat{p}(\mathbf{x}, \omega)=\int \exp (-j \omega t) p(\mathbf{x}, t) \mathrm{d} t$. In the space-frequency domain the equation of motion and the stress-strain relation thus become $\rho\left(j \omega+v_{k}^{0} \partial_{k}\right) \hat{v}_{i}+\partial_{i} \hat{p}=0$ and $\kappa\left(j \omega+v_{k}^{0} \partial_{k}\right) \hat{p}+\partial_{i} \hat{v}_{i}=\hat{q}$, respectively.

We introduce two independent acoustic states, which will be distinguished by subscripts $A$ and $B$, and consider the following combination of wave fields in both states: $\hat{p}_{A}^{*} \hat{v}_{i, B}$ $+\hat{v}_{i, A}^{*} \hat{B}_{B}$, where the asterisk denotes complex conjugation. In the following we assume that the medium parameters and flow velocities in both states are identical; only the sources and wave fields are different (but a more general derivation is possible ${ }^{26}$ ). The correlation-type reciprocity theorem is obtained by applying the differential operator $\partial_{i}$, according to $\partial_{i}\left\{\hat{p}_{A}^{*} \hat{v}_{i, B}+\hat{v}_{i, A}^{*} \hat{p}_{B}\right\}$, substituting the equation of motion and the stress-strain relation for states $A$ and $B$, integrating the result over a spatial domain $V$ with boundary $S$ and outward pointing normal vector $\mathbf{n}$ $=\left(n_{1}, n_{2}, n_{3}\right)$ and applying the theorem of Gauss. This gives

$$
\int_{V}\left\{\hat{q}_{A}^{*} \hat{p}_{B}+\hat{p}_{A}^{*} \hat{q}_{B}\right\} \mathrm{d}^{3} \mathbf{x}=\oint_{S}\left\{\hat{p}_{A}^{*} \hat{v}_{i, B}+\hat{v}_{i, A}^{*} \hat{p}_{B}\right\} n_{i} \mathrm{~d}^{2} \mathbf{x}+\int_{V} v_{k}^{0}\left\{\kappa \partial_{k}\left(\hat{p}_{A}^{*} \hat{p}_{B}\right)+\rho \partial_{k}\left(\hat{v}_{i, A}^{*} \hat{v}_{i, B}\right)\right\} \mathrm{d}^{3} \mathbf{x} .
$$

This relation is independent of the choice of $S$; moreover, the medium and flow velocity can be inhomogeneous inside as well as outside $S$. In comparison with the convolution-type reciprocity theorem, Eq. (1) is remarkably simple. The convolution-type theorem can only be simplified to a form similar to Eq. (1) by choosing opposite flow velocities in the two states. ${ }^{26-29}$ In the correlation-type theorem of Eq. (1) the flow velocities in both states are identical.

Next we choose impulsive point sources in both states, according to $\hat{q}_{A}(\mathbf{x}, \omega)=\delta(\mathbf{x}$ $\left.-\mathbf{x}_{A}\right)$ and $\hat{q}_{B}(\mathbf{x}, \omega)=\delta\left(\mathbf{x}-\mathbf{x}_{B}\right)$, with $\mathbf{x}_{A}$ and $\mathbf{x}_{B}$ both in $V$. The wave field in state $A$ can thus be expressed in terms of a Green's function, according to

$$
\begin{aligned}
& \hat{p}_{A}(\mathbf{x}, \omega)=\hat{G}^{p, q}\left(\mathbf{x}, \mathbf{x}_{A}, \omega\right), \\
& \hat{v}_{i, A}(\mathbf{x}, \omega)=\hat{G}_{i}^{v, q}\left(\mathbf{x}, \mathbf{x}_{A}, \omega\right) .
\end{aligned}
$$

The superscripts refer to the observed wave field quantity at $\mathbf{x}$ and the source type at $\mathbf{x}_{A}$, respectively. Similar expressions hold for the wave field in state $B$. Substitution into Eq. (1) gives

$$
\hat{G}^{p, q}\left(\mathbf{x}_{A}, \mathbf{x}_{B}, \omega\right)+\left\{\hat{G}^{p, q}\left(\mathbf{x}_{B}, \mathbf{x}_{A}, \omega\right)\right\}^{*}=\sum_{n=1}^{4} I_{n},
$$

where

$$
\begin{gathered}
I_{1}=\oint_{S}\left\{\hat{G}^{p, q}\left(\mathbf{x}, \mathbf{x}_{A}, \omega\right)\right\}^{*} \hat{G}_{i}^{v, q}\left(\mathbf{x}, \mathbf{x}_{B}, \omega\right) n_{i} \mathrm{~d}^{2} \mathbf{x}, \\
I_{2}=\oint_{S}\left\{\hat{G}_{i}^{v, q}\left(\mathbf{x}, \mathbf{x}_{A}, \omega\right)\right\}^{*} \hat{G}^{p, q}\left(\mathbf{x}, \mathbf{x}_{B}, \omega\right) n_{i} \mathrm{~d}^{2} \mathbf{x}, \\
I_{3}=\int_{V} v_{k}^{0} \kappa \partial_{k}\left[\left\{\hat{G}^{p, q}\left(\mathbf{x}, \mathbf{x}_{A}, \omega\right)\right\}^{*} \hat{G}^{p, q}\left(\mathbf{x}, \mathbf{x}_{B}, \omega\right)\right] \mathrm{d}^{3} \mathbf{x},
\end{gathered}
$$




$$
I_{4}=\int_{V} v_{k}^{0} \rho \partial_{k}\left[\left\{\hat{G}_{i}^{v, q}\left(\mathbf{x}, \mathbf{x}_{A}, \omega\right)\right\}^{*} \hat{G}_{i}^{v, q}\left(\mathbf{x}, \mathbf{x}_{B}, \omega\right)\right] \mathrm{d}^{3} \mathbf{x} .
$$

Equations (4)-(8) show how the Green's function in a medium with flow can, in principle, be obtained from cross correlations of Green's functions in the same medium. However, application of these equations requires the measurements of different types of Green's function. In the following we make a number of approximations which make these expressions suited for practical applications.

First we assume that the medium at and outside $S$ is homogeneous, so that the Green's functions in $I_{1}$ and $I_{2}$ represent outgoing waves at $S$. Moreover, we assume that the flow velocity at $S$ is small in comparison with the propagation velocity $c$, i.e., $\left|v_{k}^{0} n_{k}\right| / c \ll 1$. We express the Green's function $\hat{G}_{i}^{v, q}$ in terms of $\hat{G}^{p, q}$ using the approximation $\hat{G}_{i}^{v, q} n_{i} \approx(1 / \rho c) \hat{G}^{p, q}$. This is the high-frequency approximation for a normally outward propagating ray in a nonflowing medium. It involves an amplitude error for non-normal outward propagating rays in a flowing medium, but it handles the phase correctly (in the high-frequency regime). By using this approximation we avoid the need of determining the inhomogeneous propagation and flow models, tracing the rays and computing the propagation angles at $S$. With this approximation we find $^{30}$

$$
I_{1} \approx I_{2} \approx \frac{1}{\rho c} \oint_{S} \hat{G}^{*}\left(\mathbf{x}, \mathbf{x}_{A}, \omega\right) \hat{G}\left(\mathbf{x}, \mathbf{x}_{B}, \omega\right) \mathrm{d}^{2} \mathbf{x} .
$$

Here and in the following $\hat{G}$ stands for $\hat{G}^{p, q}$. To show that $I_{3}$ and $I_{4}$ are small, we assume that the spatial variations of the medium parameters (as well as those of the flow velocity) are small in comparison with those of the wave field. Using the theorem of Gauss and $\kappa=1 / \rho c^{2}$ we may thus rewrite $I_{3}$ as

$$
I_{3} \approx \frac{1}{\rho c} \oint_{S} \hat{G}^{*}\left(\mathbf{x}, \mathbf{x}_{A}, \omega\right) \hat{G}\left(\mathbf{x}, \mathbf{x}_{B}, \omega\right) \frac{v_{k}^{0} n_{k}}{c} \mathrm{~d}^{2} \mathbf{x} .
$$

Using the aforementioned assumption $\left|v_{k}^{0} n_{k}\right| / c \ll 1$ we thus find $I_{3} \ll I_{1}$. In a similar way we find $I_{4} \ll I_{1}$. In the following we replace the right-hand side of Eq. (4) by $2 I_{1}$, with $I_{1}$ approximated by Eq. (9).

Next we interchange the source and receiver coordinates in the Green's functions. According to the flow reversal theorem ${ }^{26-29}$ this is allowed if we simultaneously revert the flow direction, i.e., if we replace $v_{k}^{0}(\mathbf{x})$ by $-v_{k}^{0}(\mathbf{x})$. We apply this to all Green's functions in Eq. (4), with the right-hand side approximated by $2 I_{1}$, hence

$$
\hat{G}\left(\mathbf{x}_{B}, \mathbf{x}_{A}, \omega\right)+\hat{G}^{*}\left(\mathbf{x}_{A}, \mathbf{x}_{B}, \omega\right) \approx \frac{2}{\rho c} \oint_{S} \hat{G}^{*}\left(\mathbf{x}_{A}, \mathbf{x}, \omega\right) \hat{G}\left(\mathbf{x}_{B}, \mathbf{x}, \omega\right) \mathrm{d}^{2} \mathbf{x},
$$

where all Green's functions are now defined in a medium with flow velocity $-v_{k}^{0}(\mathbf{x})$. The minus sign is not important; what matters is that the flow velocity is the same for all Green's functions in this equation. From here onward we define $w_{k}^{0}(\mathbf{x})=-v_{k}^{0}(\mathbf{x})$ as the actual flow velocity. Hence, Eq. (11) applies to the actual situation and, with hindsight, Eqs. (4)-(10) apply to the situation with the reversed flow velocity $-w_{k}^{0}(\mathbf{x})$.

Applying an inverse Fourier transform to Eq. (11) yields

$$
G\left(\mathbf{x}_{B}, \mathbf{x}_{A}, t\right)+G\left(\mathbf{x}_{A}, \mathbf{x}_{B},-t\right) \approx \frac{2}{\rho c} \oint_{S} G\left(\mathbf{x}_{A}, \mathbf{x},-t\right) * G\left(\mathbf{x}_{B}, \mathbf{x}, t\right) \mathrm{d}^{2} \mathbf{x},
$$

where the asterisk denotes temporal convolution. The right-hand side represents an integral of cross correlations of observations of the acoustic pressure in a moving medium at $\mathbf{x}_{A}$ and $\mathbf{x}_{B}$, 


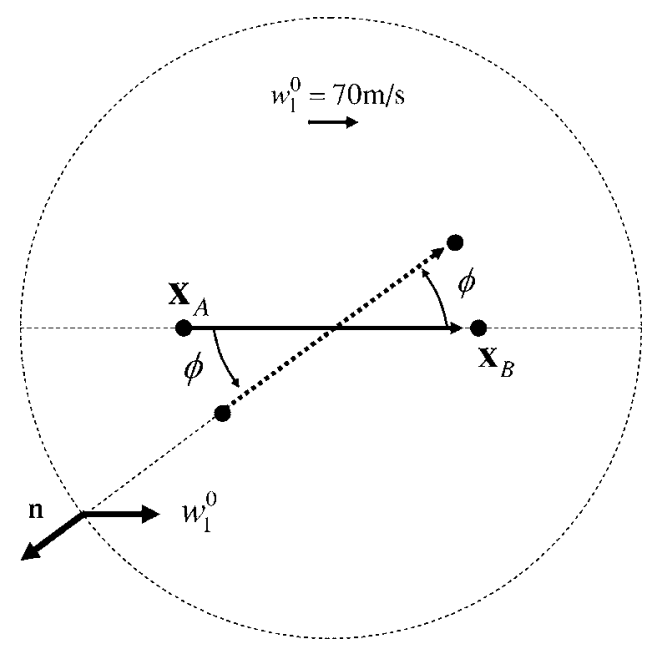

Fig. 1. Two receivers at $\mathbf{x}_{A}$ and $\mathbf{x}_{B}$ in a moving medium with constant flow velocity. The receivers are surrounded by 360 noise sources on a circle.

respectively, due to impulsive sources of volume injection rate at $\mathbf{x}$ on $S$; the integration takes place along the source coordinate $\mathbf{x}$. The left-hand side is the superposition of the response from $\mathbf{x}_{A}$ to $\mathbf{x}_{B}$ and the time-reversed response from $\mathbf{x}_{B}$ to $\mathbf{x}_{A}$. Note the similarity with the expressions for the situation of a nonmoving medium. ${ }^{13-16,30,31}$ However, unlike for the situation of a nonmoving medium, our result is asymmetric in time. $G\left(\mathbf{x}_{B}, \mathbf{x}_{A}, t\right)$ is obtained by taking the causal part of the left-hand side of Eq. (12), $G\left(\mathbf{x}_{A}, \mathbf{x}_{B}, t\right)$ by time-reverting the acausal part.

Until now we assumed that the sources on $S$ are impulsive point sources, of which the responses are measured independently. Let us now consider noise sources $N(\mathbf{x}, t)$ that act simultaneously for all $\mathbf{x}$ on $S$. For the observed wave field at $\mathbf{x}_{A}$ we write $p^{\text {obs }}\left(\mathbf{x}_{A}, t\right)$ $=\oint_{S} G\left(\mathbf{x}_{A}, \mathbf{x}, t\right) * N(\mathbf{x}, t) \mathrm{d}^{2} \mathbf{x}$; a similar expression holds for the observed wave field at $\mathbf{x}_{B}$. We assume that any two noise sources $N(\mathbf{x}, t)$ and $N\left(\mathbf{x}^{\prime}, t\right)$ with $\mathbf{x} \neq \mathbf{x}^{\prime}$ are uncorrelated and that their autocorrelation $C(t)$ is independent of $\mathbf{x}$. Hence, we assume that the source distribution on $S$ obeys the relation $\left\langle N(\mathbf{x},-t) * N\left(\mathbf{x}^{\prime}, t\right)\right\rangle=\delta\left(\mathbf{x}-\mathbf{x}^{\prime}\right) C(t)$, where $\langle\cdot\rangle$ denotes a spatial ensemble average. ${ }^{6,12-16}$ Equation (12) can thus be rewritten as

$$
\left\{G\left(\mathbf{x}_{B}, \mathbf{x}_{A}, t\right)+G\left(\mathbf{x}_{A}, \mathbf{x}_{B},-t\right)\right\} * C(t) \approx \frac{2}{\rho c}\left\langle p^{\mathrm{obs}}\left(\mathbf{x}_{A},-t\right) * p^{\mathrm{obs}}\left(\mathbf{x}_{B}, t\right)\right\rangle .
$$

According to this equation the cross correlation of the observed noise fields at $\mathbf{x}_{A}$ and $\mathbf{x}_{B}$ in a moving medium yields the Green's function from $\mathbf{x}_{A}$ to $\mathbf{x}_{B}$ plus the time-reversed Green's function from $\mathbf{x}_{B}$ to $\mathbf{x}_{A}$, convolved with the autocorrelation of the noise sources. Note the resemblance with the retrieval of the Green's function in a diffuse wave field in a nonmoving medium. ${ }^{4-16}$ Again the main difference is the temporal asymmetry of the correlation result in a moving medium versus the symmetry of that in a nonmoving medium.

\section{Numerical example}

We illustrate Eq. (13) with a 2-D numerical example. Consider a homogeneous medium with propagation velocity $c=350 \mathrm{~m} / \mathrm{s}$ and a constant flow in the $x_{1}$ direction, with flow velocity $w_{1}^{0}=70 \mathrm{~m} / \mathrm{s}$ (see Fig. 1). Hence, the Mach number, defined as $\mathrm{M}=w_{1}^{0} / c$, equals 0.2 . Following a similar derivation as for the 3-D situation ${ }^{28}$ we obtain for the 2-D Green's function $\hat{G}\left(\mathbf{x}, \mathbf{x}_{A}, \omega\right)=\rho\left(j \omega+w_{1}^{0} \partial_{1}\right) \hat{\mathcal{G}}\left(\mathbf{x}, \mathbf{x}_{A}, \omega\right)$, with 

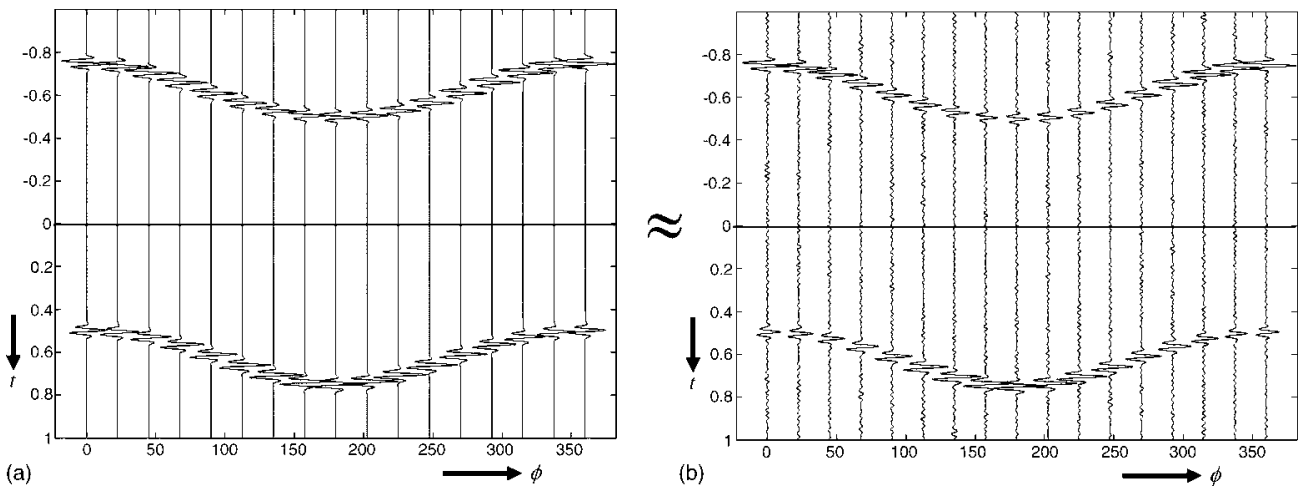

Fig. 2. (a) Left- and (b) right-hand side of Eq. (13) for different values of $\phi$ (the angle between the flow velocity and the line through $\mathbf{x}_{A}$ and $\mathbf{x}_{B}$, see Fig. 1).

$$
\hat{\mathcal{G}}=-\frac{j \exp \left(j \omega \mathrm{M}\left(x_{1}-x_{1, A}\right) / c\left(1-\mathrm{M}^{2}\right)\right)}{4 \sqrt{1-\mathrm{M}^{2}}} \mathrm{H}_{0}^{(2)}\left(\frac{\omega R}{c\left(1-\mathrm{M}^{2}\right)}\right),
$$

where $R=\sqrt{\left(x_{1}-x_{1, A}\right)^{2}+\left(1-\mathrm{M}^{2}\right)\left(x_{2}-x_{2, A}\right)^{2}}$ and $\mathrm{H}_{0}^{(2)}$ is the zeroth-order Hankel function of the second kind. Using this expression we model the response of 360 uncorrelated noise sources on a circle with a radius of $470 \mathrm{~m}$ (the noise is filtered around a central frequency of $30 \mathrm{~Hz}$ ). We consider two receivers at $\mathbf{x}_{A}$ and $\mathbf{x}_{B}$, separated by a distance $d=210 \mathrm{~m}$, each registering $9600 \mathrm{~s}$ of noise. In the first instance the line through $\mathbf{x}_{A}$ and $\mathbf{x}_{B}$ is aligned with the flow velocity, hence $\phi$ in Fig. 1 equals zero. The cross correlation of the noise registrations, i.e., the right-hand side of Eq. (13), is represented by the first trace in Fig. 2(b) (at $\phi=0$ ). The numerical experiment is repeated for different angles $\phi$ between the flow velocity and the line through $\mathbf{x}_{A}$ and $\mathbf{x}_{B}$; the cross-correlation results are represented by the other traces in Fig. 2(b). The Green's functions convolved with $C(t)$ in the left-hand side of Eq. (13) are shown for the same range of angles $\phi$ in Fig. 2(a). For $\phi=0$ the traveltime of the causal and acausal Green's functions are given by $d / c(1+\mathrm{M})=210 / 420=0.5 \mathrm{~s}$ and $-d / c(1-\mathrm{M})=-210 / 280=-0.75 \mathrm{~s}$, respectively. For $\phi=90^{\circ}$ the travel times are $\pm d / c \sqrt{1-\mathrm{M}^{2}}= \pm 0.612 \mathrm{~s}$. Note that the travel times of the cross-correlation results accurately match those of the Green's functions for all angles $\phi$. The amplitudes of the Green's functions are less accurately recovered by the cross-correlation procedure (see Fig. 3). The amplitude errors are explained as follows. The main contributions to the integrals come from those sources on the circle where the line through $\mathbf{x}_{A}$ and $\mathbf{x}_{B}$ intersects the circle ${ }^{12,30}$ (see Fig. 1). Ignoring $I_{3}$ and $I_{4}$ with respect to $I_{1}$ and $I_{2}$ introduces a relative amplitude error in the order of $-v_{k}^{0} n_{k} / c=w_{1}^{0} n_{1} / c$, with $n_{1}=-\cos \phi$ (see Fig. 1). Evaluated as a function of $\phi$ we thus find for the relative amplitude error $w_{1}^{0} n_{1} / c=-0.2 \cos \phi$, which is approximately what we observe in Fig. 3.

\section{Conclusion}

We have shown that the nonreciprocal Green's function in a moving medium can be recovered from cross correlations of impulse responses [Eq. (12)] or noise measurements [Eq. (13)] at two receivers. The sources are assumed to be distributed along an arbitrary surface enclosing the two receivers. Unlike in the situation of a nonmoving medium, the cross-correlation result is asymmetric in time. The theory holds for a lossless arbitrary inhomogeneous medium with stationary inhomogeneous flow. The main underlying assumptions (in addition to those for a nonmoving medium) are that the spatial variations of the flow velocity are small in comparison with those of the wave field and that the flow velocity is small in comparison with the propagation velocity (small Mach number). The cross-correlation method accurately recovers the travel 


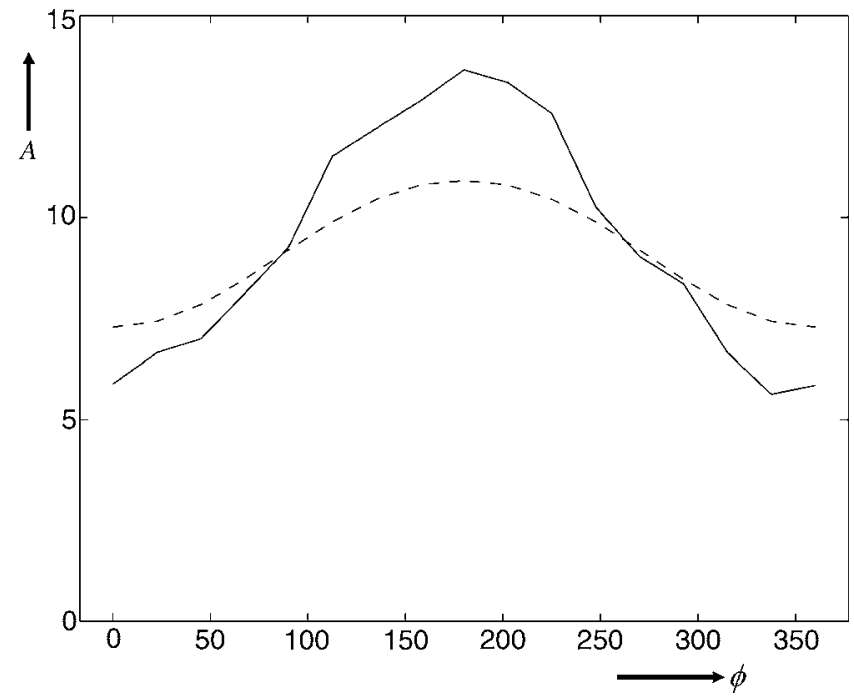

Fig. 3. Maximum amplitudes of the causal part of Figs. 2(a) (dashed) and 2(b) (solid).

times of the nonreciprocal Green's function. When the autocorrelation of the sources is known, the amplitudes are recovered with relative errors that are in the order of the Mach number. This error is negligible in comparison with the amplitude error that occurs when the sources are unknown. Hence, for practical situations (unknown source amplitudes, irregular source distribution, etc.), the accuracy of the retrieved nonreciprocal Green's function in a moving medium is of the same order as that of the retrieved reciprocal Green's function in a nonmoving medium.

\section{References}

${ }^{1}$ J. F. Claerbout, "Synthesis of a layered medium from its acoustic transmission response," Geophysics 33,

264-269 (1968).

${ }^{2}$ T. L. Duvall, S. M. Jefferies, J. W. Harvey, and M. A. Pomerantz, “Time-distance helioseismology,” Nature (London) 362, 430-432 (1993).

${ }^{3} \mathrm{~J}$. Rickett and J. Claerbout, "Acoustic daylight imaging via spectral factorization: Helioseismology and reservoir monitoring," The Leading Edge 18(8), 957-960 (1999).

${ }^{4}$ R. L. Weaver and O. I. Lobkis, "Ultrasonics without a source: Thermal fluctuation correlations at MHz frequencies," Phys. Rev. Lett. 87, 134301-1-134301-4 (2001).

${ }^{5}$ M. Campillo and A. Paul, "Long-range correlations in the diffuse seismic coda," Science 299, 547-549 (2003).

${ }^{6} \mathrm{P}$. Roux, W. A. Kuperman, and the NPAL Group, "Extracting coherent wave fronts from acoustic ambient noise in the ocean," J. Acoust. Soc. Am. 116, 1995-2003 (2004).

${ }^{7}$ N. M. Shapiro, M. Campillo, L. Stehly, and M. H. Ritzwoller, "High-resolution surface-wave tomography from ambient seismic noise," Science 307, 1615-1618 (2005).

${ }^{8}$ O. I. Lobkis and R. L. Weaver, "On the emergence of the Green's function in the correlations of a diffuse field," J. Acoust. Soc. Am. 110, 3011-3017 (2001).

${ }^{9}$ R. L. Weaver and O. I. Lobkis, "On the emergence of the Green's function in the correlations of a diffuse field: pulse-echo using thermal phonons," Ultrasonics 40, 435-439 (2002).

${ }^{10}$ B. A. van Tiggelen, "Green function retrieval and time reversal in a disordered world," Phys. Rev. Lett. 91, 243904-1-243904-4 (2003).

${ }^{11}$ A. E. Malcolm, J. A. Scales, and B. A. van Tiggelen, "Extracting the Green function from diffuse, equipartitioned waves," Phys. Rev. E 70, 015601(R)-1-015601(R)-4 (2004).

${ }^{12} \mathrm{R}$. Snieder, "Extracting the Green's function from the correlation of coda waves: A derivation based on stationary phase," Phys. Rev. E 69, 046610-1-046610-8 (2004).

${ }^{13} \mathrm{~K}$. Wapenaar, D. Draganov, J. Thorbecke, and J. Fokkema, "Theory of acoustic daylight imaging revisited," in 72nd Annual Meeting of the Society of Exploration Geophysicists (SEG), Salt Lake City, 2002, edited by Mike H. Powers (SEG, Tulsa, 2002), pp. 2269-2272.

${ }^{14} \mathrm{~K}$. Wapenaar, "Retrieving the elastodynamic Green's function of an arbitrary inhomogeneous medium by cross correlation,” Phys. Rev. Lett. 93, 254301-1-254301-4 (2004).

${ }^{15}$ R. L. Weaver and O. I. Lobkis, "Diffuse fields in open systems and the emergence of the Green's function (L)," 
J. Acoust. Soc. Am. 116, 2731-2734 (2004).

${ }^{16}$ A. Derode, E. Larose, M. Tanter, J. de Rosny, A. Tourin, M. Campillo, and M. Fink, "Recovering the Green's function from field-field correlations in an open scattering medium (L)," J. Acoust. Soc. Am. 113, 2973-2976 (2003).

${ }^{17}$ M. Fink, "Time reversed acoustics," Phys. Today 50, 34-40 (1997).

${ }^{18}$ P. Roux, K. G. Sabra, W. A. Kuperman, and A. Roux, “Ambient noise cross correlation in free space: Theoretical approach," J. Acoust. Soc. Am. 117, 79-84 (2005).

${ }^{19}$ E. Slob, D. Draganov, and K. Wapenaar, "GPR without a source," in Eleventh International Conference on Ground Penetrating Radar, Columbus, Ohio, 2006, edited by C.-C. Chen and J. Daniels (2006).

${ }^{20}$ D. R. Dowling, "Phase-conjugate array focusing in a moving medium," J. Acoust. Soc. Am. 94, 1716-1718 (1993).

${ }^{21}$ P. Roux, W. A. Kuperman, W. S. Hodgkiss, H. C. Song, T. Akal, and M. Stevenson, “A nonreciprocal implementation of time reversal in the ocean," J. Acoust. Soc. Am. 116, 1009-1015 (2004).

${ }^{22}$ A. T. de Hoop, "Time-domain reciprocity theorems for acoustic wave fields in fluids with relaxation," J. Acoust. Soc. Am. 84, 1877-1882 (1988)

${ }^{23}$ J. T. Fokkema and P. M. van den Berg, Seismic Applications of Acoustic Reciprocity (Elsevier, Amsterdam, 1993).

${ }^{24} \mathrm{~N}$. N. Bojarski, "Generalized reaction principles and reciprocity theorems for the wave equations, and the relationship between the time-advanced and time-retarded fields," J. Acoust. Soc. Am. 74, 281-285 (1983).

${ }^{25}$ P. M. Morse and K. U. Ingard, Theoretical Acoustics (McGraw-Hill, New York, 1968).

${ }^{26} \mathrm{~K}$. Wapenaar and J. Fokkema, "Reciprocity theorems for diffusion, flow and waves," J. Appl. Mech. 71, $145-150$ (2004)

${ }^{27}$ L. M. Lyamshev, "On some integral relationships in acoustics of moving medium,” Dokl. Akad. Nauk SSSR 138, 575-578 (1961).

${ }^{28}$ L. M. Brekhovskikh and O. A. Godin, Acoustics of Layered Media II. Point Sources and Bounded Beams (Springer, Berlin, 1992).

${ }^{29}$ B. P. Belinskiy, On Some General Mathematical Properties of the System: Elastic Plate-Acoustic Medium (World Scientific, Singapore 2001), pp. 193-218.

${ }^{30} \mathrm{~K}$. Wapenaar, J. Fokkema, and R. Snieder, "Retrieving the Green's function in an open system by crosscorrelation: a comparison of approaches (L)," J. Acoust. Soc. Am. 118, 2783-2786 (2005).

${ }^{31}$ D.-J. van Manen, J. O. A. Robertsson, and A. Curtis, "Modeling of wave propagation in inhomogeneous media," Phys. Rev. Lett. 94, 164301-1-164301-4 (2005). 